\title{
Highly ionized region surrounding SN Refsdal revealed by MUSE
}

\author{
W. Karman ${ }^{1}$, C. Grillo ${ }^{2}$, I. Balestra ${ }^{3,4}$, P. Rosati ${ }^{5}$, K. I. Caputi ${ }^{1}$, E. Di Teodoro ${ }^{6}$, F. Fraternali ${ }^{1,6}$, R. Gavazzi ${ }^{7}$, \\ A. Mercurio ${ }^{8}$, J. X. Prochaska ${ }^{9}$, S. Rodney ${ }^{10,11}$, and T. Treu ${ }^{12}$ \\ ${ }^{1}$ Kapteyn Astronomical Institute, University of Groningen, Postbus 800, 9700 AV Groningen, The Netherlands \\ e-mail: karman@astro.rug.nl \\ ${ }^{2}$ Dark Cosmology Centre, Niels Bohr Institute, University of Copenhagen, Juliane Maries Vej 30, 2100 Copenhagen, Denmark \\ 3 INAF-Osservatorio Astronomico di Trieste, via G. B. Tiepolo 11, 34143 Trieste, Italy \\ ${ }^{4}$ University Observatory Munich, Scheinerstrasse 1, 81679 Munich, Germany \\ 5 Dipartimento di Fisica e Scienze della Terra, Università degli Studi di Ferrara, via Saragat 1, 44122 Ferrara, Italy \\ 6 Department of Physics and Astronomy, University of Bologna, 6/2, Viale Berti Pichat, 40127 Bologna, Italy \\ 7 Institut d'Astrophysique de Paris, UMR7095 CNRS-Université Pierre et Marie Curie, 98bis Bd Arago, 75014 Paris, France \\ 8 INAF-Osservatorio Astronomico di Capodimonte, via Moiariello 16, 80131 Napoli, Italy \\ 9 Department of Astronomy and Astrophysics, UCO/Lick Observatory, University of California, Santa Cruz, CA 95064, USA \\ 10 Department of Physics and Astronomy, University of South Carolina, 712 Main St., Columbia, SC 29208, USA \\ 11 Department of Physics and Astronomy, The Johns Hopkins University, Baltimore, MD 21218, USA \\ 12 Department of Physics and Astronomy, University of California, Los Angeles, CA 90095, USA
}

Received 24 September 2015 / Accepted 9 November 2015

\section{ABSTRACT}

\begin{abstract}
Supernova (SN) Refsdal is the first multiply imaged, highly magnified, and spatially resolved SN ever observed. The SN exploded in a highly magnified spiral galaxy at $z=1.49$ behind the Frontier Fields cluster MACS1149, and provides a unique opportunity to study the environment of $\mathrm{SNe}$ at high $z$. We exploit the time delay between multiple images to determine the properties of the $\mathrm{SN}$ and its environment before, during, and after the SN exploded. We use the integral-field spectrograph MUSE on the VLT to simultaneously target all observed and model-predicted positions of SN Refsdal. We find Mg II emission at all positions of SN Refsdal, accompanied by weak Fe II* emission at two positions. The measured ratios of [O II] to Mg II emission of 10-20 indicate a high degree of ionization with low metallicity. Because the same high degree of ionization is found in all images, and our spatial resolution is too coarse to resolve the region of influence of SN Refsdal, we conclude that this high degree of ionization has been produced by previous SNe or a young and hot stellar population. We find no variability of the [O II] line over a period of 57 days. This suggests that there is no variation in the [O II] luminosity of the $\mathrm{SN}$ over this period, or that the $\mathrm{SN}$ has a small contribution to the integrated [O II] emission over the scale resolved by our observations.
\end{abstract}

Key words. galaxies: high-redshift - galaxies: star formation - galaxies: ISM - galaxies: evolution - gravitational lensing: strong techniques: spectroscopic

\section{Introduction}

Supernova (SN) Refsdal (Kelly et al. 2015) is the first observed lensed SN resolved into multiple images. Being hosted by a spiral galaxy (Sp1149) that is multiply imaged itself, the discovery presents the possibility of studying the environment of SN Refsdal at three different epochs, with lensing affording spatial scales of $\sim 0.7 \mathrm{kpc}$. Preliminary strong-lensing models (e.g. Oguri 2015; Sharon \& Johnson 2015; Diego et al. 2015) predicted that we are now witnessing the second appearance of the $\mathrm{SN}$. Therefore, we are able to study the environment of the SN before, during, and after its explosion.

Although SN Refsdal is a very interesting object, determining the properties of its environment could be even more important from the point of view of galaxy evolution. Galaxy growth is regulated by feedback and outflows, which are ascribed to energetic processes such as $\mathrm{SNe}$ and active galactic nuclei. The necessity of feedback is widely acknowledged, but the detailed processes that regulate it are still under debate. Feedback of SNe becomes more efficient when the surrounding medium has already been heated and diluted by young and hot stars (e.g. Stinson et al. 2013; Hopkins et al. 2014; Artale et al. 2015; Kim \& Ostriker 2015; Martizzi et al. 2015; Vasiliev et al. 2015), and observations of the properties of the interstellar medium (ISM) surrounding $\mathrm{SNe}$ are crucial to confirm this heating, especially at high redshift where feedback is strongest. The resolution and luminosity required to study the environment of $\mathrm{SNe}$ at high redshift can currently only be achieved through gravitational lensing.

Given its recent discovery, the environment of SN Refsdal has not yet been studied in detail, but its host galaxy was the focus of several works. Sp1149 (Smith et al. 2009a), located behind the Frontier Fields cluster MACS1149 $(z=0.542)$, is a spiral galaxy at $z=1.49$ with many star-forming clumps in its arms, and a large magnification (Zitrin \& Broadhurst 2009). Yuan et al. $(2011,2015)$ found a steep metallicity gradient in Sp1149, measuring a low metallicity $\left(Z \lesssim 0.3 Z_{\odot}\right)$ at the position of SN Refsdal. Active, widespread star formation (SF) was shown by rest-frame UV observations (Smith et al. 2009a), and through $\mathrm{H} \alpha$ detection (Livermore et al. 2012, 2015) at a rate of a few $M_{\odot} \mathrm{yr}^{-1}$. The size and age of the clumps increase inwards, with a young population of stars at the position of SN Refsdal (Adamo et al. 2013).

The environment of SN Refsdal can be studied through the presence of certain emission lines. In the optical regime, $\mathrm{Mg}$ II 


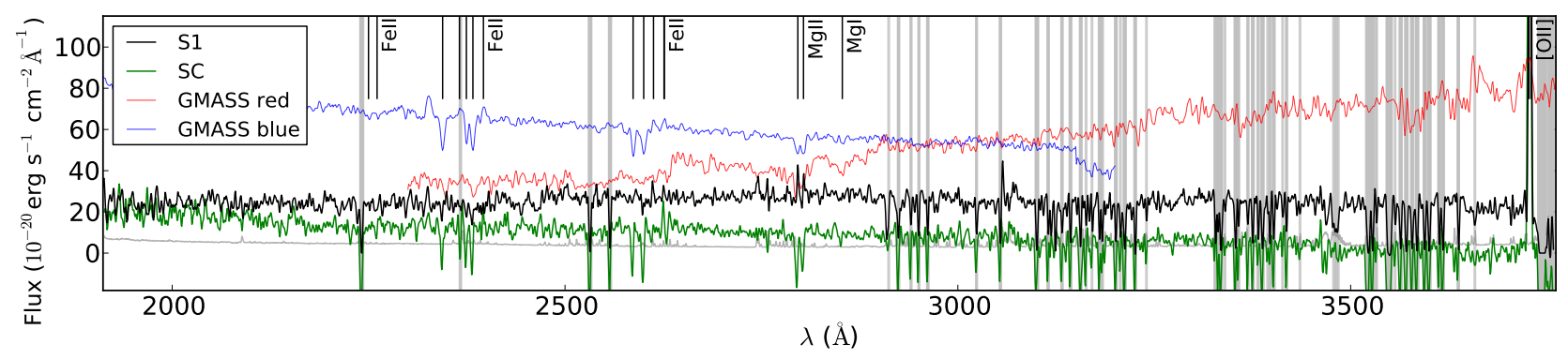

Fig. 1. Rest-frame spectrum of Sp1149 at the position S1 and its centre (SC), which have been smoothed over 9 pixels for illustrative purposes. For comparison, the red and blue composite GMASS spectra (Kurk et al. 2013) are also shown, which are normalized to the Sp1149 spectrum at $3000 \AA$. To avoid confusion, we added an offset of $(-25,25) \times 10^{-20} \mathrm{erg} \mathrm{s}^{-1} \mathrm{~cm}^{-2} \AA^{-1}$ to the central spectrum and the GMASS spectra, respectively. The error estimates are shown by the grey line, while the vertical grey bands are located at wavelengths with significant skyline contamination. The short black vertical lines show the wavelength of important $\mathrm{Fe}$ and $\mathrm{Mg}$ lines.

is frequently used, as it is detectable from the ground over a wide redshift range. Weiner et al. (2009) were the first to report $\mathrm{Mg}$ II emission lines in galaxies at $z>1$, and these lines have been found in several studies since (Pettini et al. 2010; Rubin et al. 2011; Martin et al. 2012; Erb et al. 2012; Kornei et al. 2013; Rigby et al. 2014; Tang et al. 2014; Ben Zhu et al. 2015). The origin of Mg II emission can either be H II regions (see Kinney et al. 1993), or resonant scattering in outflowing gas (Prochaska et al. 2011). Rubin et al. (2011) found Mg II emission in a galaxy at $z=0.69$ that was clearly extended farther out than the continuum emission, in support of the latter scenario. However, Erb et al. (2012) found that the Mg II emission in their sample was more similar to $\mathrm{H}$ II regions, supporting the former.

In this paper, we study the properties of the region surrounding SN Refsdal in the highly magnified, multiply imaged spiral galaxy Sp1149 at $z=1.49$ through high-quality integral field spectroscopy at all of its observed and model-predicted multiple image positions. In Sect. 2 we present the observations, in Sect. 3 we show the results of our observations, we discuss the implications in Sect. 4, and we conclude in Sect. 5.

\section{Observations}

We observed MACS1149 with the Multi Unit Spectroscopic Explorer (MUSE, Bacon et al. 2012) instrument mounted on the Very Large Telescope at Paranal observatory. The total observation time was 6 hours of the Director's Discretionary Time (DDT), and the observations were executed in service mode (PI: C. Grillo; ID 294.A-5032). The telescope was centered at $\alpha=11: 49: 35.75, \delta=22: 23: 52.4$, such that three images of Sp1149 are within the field of view (FOV). The observations were carried out in the nights of February 14, 2015 (1 h), March 21, 2015 (4 h), and April 12, 2015 (1 h). Each hour consisted of two exposures of $1440 \mathrm{~s}$ each, such that the total exposure time adds up to $17280 \mathrm{~s}$ or $4.8 \mathrm{~h}$. The exposures were taken under clear and photometric conditions, and the seeing was $<1.1^{\prime \prime}$ in 10 out of the 12 exposures. The last hour of the observations in March was executed with a significantly worse seeing of $\sim 2^{\prime \prime}$.

The data reduction was performed using the MUSE Data Reduction Software version 1.0. The final datacube consists of pixels with a spatial scale of $0.2^{\prime \prime} \times 0.2^{\prime \prime}$ and a spectral scale of $1.25 \AA$, although we also constructed a datacube with a higher spectral sampling of $0.82 \AA$. We measured the spatial full width at half maximum (FWHM) of the final datacube from a bright star in the FOV to be 0.9", while the spectral resolution is $\sim 2.3 \AA$. This seeing corresponds to a spatial scale in the
Table 1. Observed (S1-4) and model-predicted (future: SX, past: SY) positions of SN Refsdal, the centre of Sp1149 (SC), and four starforming clumps for comparison (F1-4).

\begin{tabular}{cccccc}
\hline \hline Cl. & $\begin{array}{c}\text { RA } \\
(\mathrm{J} 2000)\end{array}$ & $\begin{array}{c}\text { Dec } \\
(\mathrm{J} 2000)\end{array}$ & $\mu^{a}$ & \multicolumn{2}{c}{$\begin{array}{c}F_{\text {OII }}^{b} \\
\left(10^{-18} \mathrm{erg} \mathrm{s}^{-1} \mathrm{~cm}^{-2}\right)\end{array}$} \\
\hline S1 & 177.39821 & 22.39563 & $13.5_{-2.2}^{+3.9}$ & $39.1 \pm 1.7$ & $2.6 \pm 0.6$ \\
S2 & 177.39771 & 22.39579 & $12.4_{-3.5}^{+6.4}$ & $29.2 \pm 1.0$ & $2.3 \pm 1.0$ \\
S3 & 177.39737 & 22.39554 & $13.4_{-3.1}^{+5.8}$ & $20.4 \pm 0.9$ & $1.6 \pm 1.1$ \\
S4 & 177.39780 & 22.39517 & $5.7_{-2.0}^{+2.4}$ & $16.3 \pm 1.2$ & $1.8 \pm 1.3$ \\
SX & 177.40012 & 22.39670 & $4.8_{-0.3}^{+0.5}$ & $15.0 \pm 1.9$ & $1.6 \pm 0.8$ \\
SY & 177.40382 & 22.40205 & $4.0_{-0.2}^{+0.2}$ & $15.2 \pm 1.7$ & $1.5 \pm 1.2$ \\
SC & 177.39702 & 22.39599 & $7.0_{-0.5}^{+0.6}$ & $56.8 \pm 1.1$ & - \\
F1 & 177.39744 & 22.39641 & $11.7_{-0.8}^{+1.3}$ & $25.8 \pm 1.5$ & - \\
F2 & 177.39883 & 22.39769 & $8.5_{-0.6}^{+0.8}$ & $26.7 \pm 1.9$ & $2.9 \pm 1.4$ \\
F3 & 177.39975 & 22.39656 & $9.4_{-0.8}^{+0.7}$ & $19.6 \pm 1.6$ & $2.1 \pm 1.0$ \\
F4 & 177.40313 & 22.40257 & $3.9_{-0.2}^{+0.1}$ & $30.5 \pm 1.8$ & $0.2 \pm 0.5$ \\
\hline
\end{tabular}

Notes. ${ }^{(a)}$ Median values of the model-predicted magnification factor (Grillo et al., in prep.); ${ }^{(b)}$ emission line flux of [O II]; ${ }^{(c)}$ emission line flux of Mg II $\lambda 2796 \AA$ corrected for stellar absorption (see Guseva et al. 2013). The emission line fluxes are determined in an $0.6^{\prime \prime}$-radius aperture, and are not corrected for magnification.

source plane of $\sim 500 \mathrm{pc}$ for a maximum magnification of $\mu=16$ or $4 \mathrm{kpc}$ for the minimum magnification $\mu=2$ in Sp1149. For details on the data reduction, see Karman et al. (2015).

We extracted individual spectra in $0.6^{\prime \prime}$-radius apertures, based on the SN coordinates from Hubble Space Telescope (HST) observations and strong-lensing-modeling predictions (S1-4, SX, SY, following Oguri 2015). We updated the modelpredicted positions of SX and SY according to our refined strong-lensing model (Grillo et al., in prep.), and show all positions and magnification factors $\mu$ in Table 1 . We find no change in our conclusions when we vary the positions within the uncertainties of our model, or by adopting positions predicted by other authors. The spectroscopic observations approximately span the rest-frame wavelength range of $1910 \AA$ through $3740 \AA$, which includes the spectral lines Fe II, Mg II, and [O II].

\section{Spectral analysis}

We show the rest-frame spectrum at $\mathrm{S} 1$ in Fig. 1, where we compare the spectrum to that in the centre of the galaxy (SC) and a blue-galaxy composite spectrum from the Galaxy Mass Assembly ultra-deep Spectroscopic Survey (GMASS, Kurk et al. 2013). The slopes of the spectra at S1 and SC are very similar, 

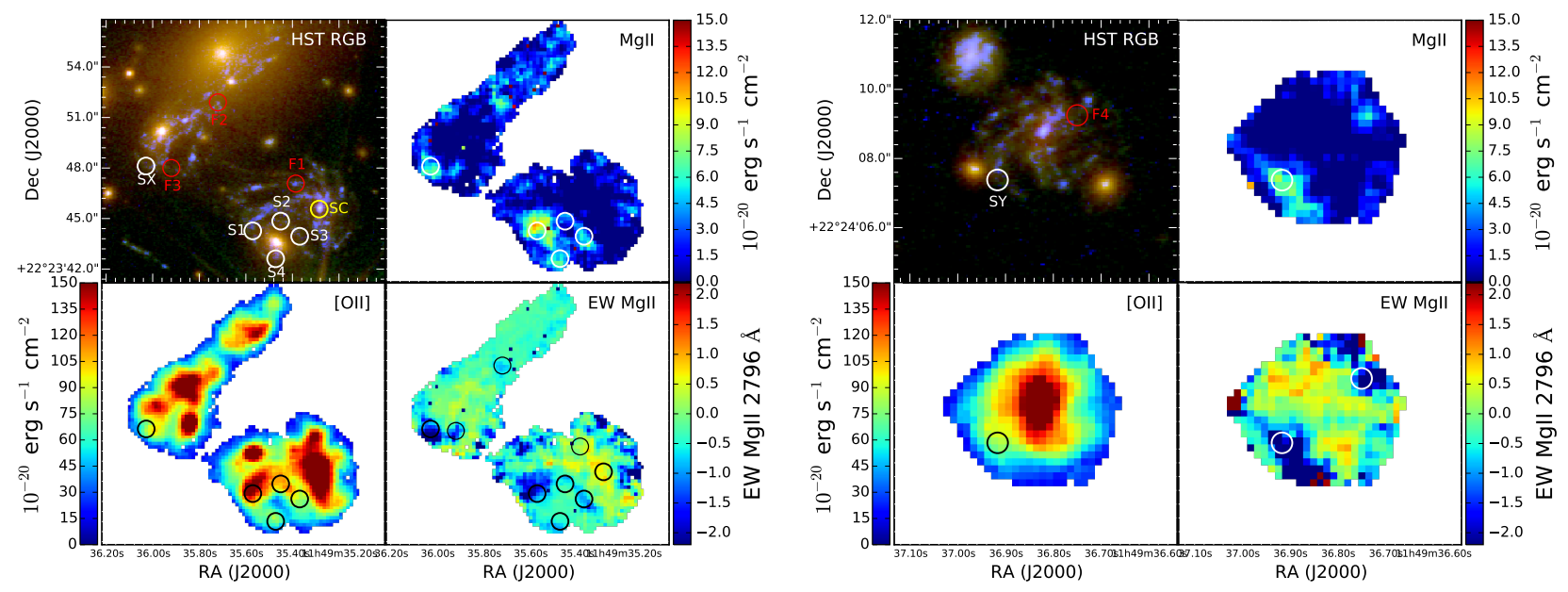

Fig. 2. Two western images (left), and the eastern image (right) of Sp1149. The two figures are divided into four panels; top left: HST RGB image with white, yellow, and red circles at the positions of SN Refsdal, the centre of the galaxy, and four star-forming clumps of Sp1149, respectively. Top right: Mg II emission line flux; bottom left: [O II] emisson-line flux; bottom right: equivalent width (EW) of Mg II $\lambda 2796 \AA$ A where a negative EW represents emission. The circles are at the same positions as the circles in the top left panel. The EW have been corrected for stellar absorption (0.5 ̊, see Guseva et al. 2013), and, for displaying purposes, the MUSE maps have been smoothed over a box of $3 \times 3$ pixels.

and comparable to the composite of GMASS. This is not surprising, as different studies have shown spread-out SF in Sp1149 (e.g. Livermore et al. 2012). The slight difference in slope between S1 and SC is probably caused by contamination of S1 from the foreground red cluster galaxy that is responsible for the Einstein cross. Looking at individual lines in the spectrum, the strong [O II] emission doublet is clear and some other lines show important differences between $\mathrm{S} 1$ and the other spectra. While the spectrum at SC and the blue composite GMASS spectrum show prominent absorption lines, these lines are significantly weaker or absent at S1. This is clearest at the Fe II $\lambda \lambda 2587$, $2600 \AA$, the $\operatorname{Mg}$ II $\lambda \lambda$ 2796, $2803 \AA$, and the $\operatorname{Mg} \mathrm{I} \lambda 2853 \AA$ absorption lines.

While $\mathrm{Mg}$ II absorption is not seen at any of the SN positions, $\mathrm{Mg}$ II emission is visible at all of them, see Figs. 2 and 3 and Table 1. Although the majority of previously reported Mg II emitters also show clear Mg II absorption, some studies found weak or no accompanying absorption (Weiner et al. 2009; Erb et al. 2012; Guseva et al. 2013; Rigby et al. 2014). In our case, the absent $\mathrm{Mg}$ II absorption, the similarly small restframe FWHM of $\sim 120$ and $\sim 140 \mathrm{~km} \mathrm{~s}^{-1}$ for Mg II and [O II], respectively, and the negligible velocity offset of $\mathrm{Mg}$ II compared to $[\mathrm{O}$ II] indicate that the $\mathrm{Mg}$ II emission originates from $\mathrm{H}$ II regions and not from resonant scattering.

In Fig. 2 we show maps of [O II] and $\mathrm{Mg}$ II emission throughout Sp1149 in all three images. While the [O II] emission is spread widely, the Mg II emission is local. The clearest $\mathrm{Mg}$ II emission we see is at position S1, while the centre of the galaxy shows strong absorption of $\mathrm{Mg}$ II. The measured lower fluxes at positions S2-4 are probably due to a lower $\mu$ when integrated around S2-4, which differs from the values of point-like images in Table 1. Since we are interested in the ISM, we corrected the equivalent width and flux of $\mathrm{Mg}$ II for stellar absorption (0.5 ̊ for young stellar populations, for details, see Guseva et al. 2013).

In Fig. 2 we see three additional regions, F2, F3, and F4, with significant flux from the $\mathrm{Mg}$ II line, which are located too far from the SN to be influenced by it. After extracting spectra of these regions, we find that the spectral properties at F2 and F3 are very similar to those of S1, see Fig. 3 for a comparison of the

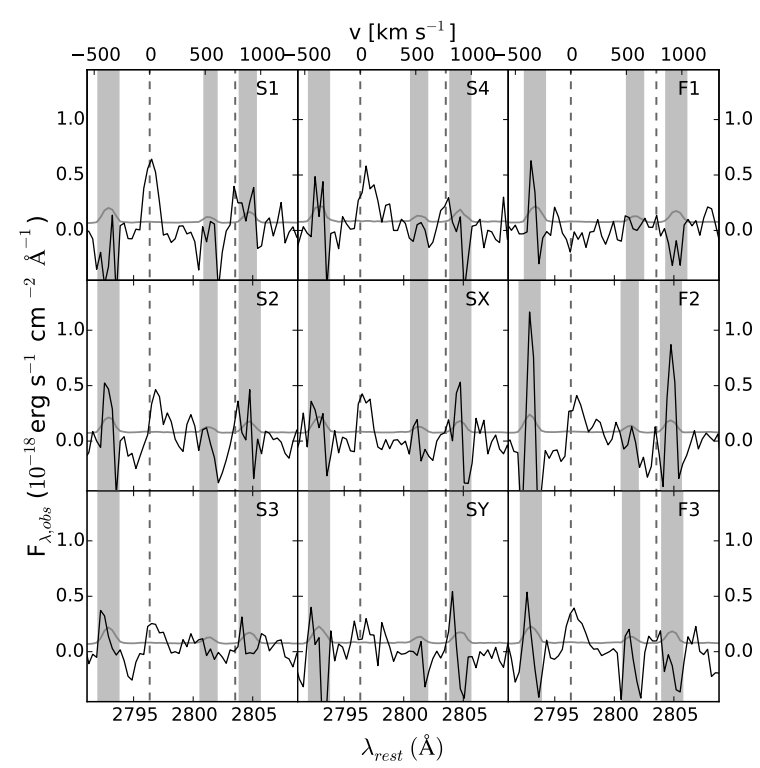

Fig. 3. Spectra around the Mg II line at all observed and modelpredicted SN positions (S1-4, SX, SY), and at three star-forming clumps (F1-3). The black solid lines show the continuum-subtracted spectra, which have been shifted to their rest frame using the [O II] doublet to determine the redshift. The vertical dashed lines are at the wavelengths of the $\mathrm{Mg}$ II doublet, the grey solid lines represent the errors, and grey vertical bands mark wavelengths with significant sky emission. The axis on the top shows the relative velocity of Mg II $\lambda 2796 \AA$ with respect to [O II].

$\mathrm{Mg}$ II lines. At F4, we find no significant detection of $\mathrm{Mg}$ II in an aperture of $0.6^{\prime \prime}$, see Table 1 . However, we find a weak emission line accompanied by more significant absorption when the size of the aperture is reduced. From the HST images we can identify F2 and F3 with two other star-forming clumps in Sp1149. Extracting spectra of other SF clumps does not reveal more Mg II emitting regions, which shows that the ionization conditions vary significantly between clumps in Sp1149. We note that although previous work showed that the properties of clumps, 
Table 2. Variability of the [O II] emission line at the four observed positions of SN Refsdal (S1 to S4).

\begin{tabular}{lccc}
\hline \hline Pos. & Feb. 14th & $\begin{array}{c}\text { Mar. 21st } \\
\left(10^{-18} \mathrm{erg} \mathrm{s}^{-1} \mathrm{~cm}^{-2}\right)\end{array}$ \\
\hline S1 & $38.2 \pm 3.9$ & $41.7 \pm 2.3$ & $40.5 \pm 5.6$ \\
S2 & $28.0 \pm 4.7$ & $28.6 \pm 2.2$ & $32.6 \pm 6.5$ \\
S3 & $22.4 \pm 4.1$ & $22.4 \pm 2.6$ & $29.2 \pm 6.3$ \\
S4 & $16.3 \pm 6.6$ & $16.3 \pm 2.1$ & $15.6 \pm 5.5$ \\
\hline
\end{tabular}

Notes. The emission line fluxes have been measured by fitting a Gaussian to the emission.

such as metallicity and stellar age, vary significantly, these variations cannot explain the observed large differences of the line ratios.

It is worth noting that we also detect $\mathrm{Mg}$ II emission at position SX, which is where the SN has not exploded yet due to a longer time delay. This indicates that the high degree of ionization is not caused by the $\mathrm{SN}$, but was already present before the SN went off. This is strengthened by the detection of additional SF clumps with $\mathrm{Mg}$ II emission. We used the ratio of [O II] and $\mathrm{Mg}$ II flux to estimate the ionization parameter $U$, following Erb et al. (2012). The emission line flux was determined by fitting a Gaussian to the spectrum, while the uncertainties were determined by repeating the calculation on 1000 mock spectra. The mock spectra were created by applying a scatter randomly picked from a normal distribution with a standard deviation equal to the error at every spectral element. We measure a ratio of 10-20 for S1-4, SX, and SY, where the main differences are due to the relatively low signal-to-noise ratio and nearby skylines. The models of Erb et al. (2012) predict an ionization parameter $-2<\log (U)<-1$ for such ratios, which is higher than that found for H II regions $(-3<\log (U)<-2$, Kinney et al. 1993), and a low metallicity in agreement with the results of Yuan et al. (2015). A metallicity even lower than used by Erb et al. (2012), that is, $Z<0.2 Z_{\odot}$, might reconcile the measured ratio with the ionization parameter found in H II regions, although we note that our measured ratio agrees with the ratios found by Guseva et al. (2013), that is, [O II]/Mg II = 5-20.

We find weaker and narrower Fe II ${ }^{*} \lambda \lambda 2612,2626 \AA$, at S1 and S2. This non-resonant emission is accompanied by Fe II absorption lines that are weaker than observed in the rest of the galaxy. The proximity of sky lines and lower magnification factors prevent us from confirming or rejecting the presence of these lines at S3, S4, SX, and SY.

The [O II] line offers an opportunity to study a possible increase in brightness of the SN. Since our data were taken in three separate months, we can compare the [O II] fluxes at intervals of 35 and 57 days. We estimate the spatial resolution of our observations to be $\sim 500-700 \mathrm{pc}$ at the positions of the SN, using the determined FWHM and the magnification factors presented in Table 1. This spatial resolution is lower than the resolution of HST and the resolved scale is much larger than the region of influence of the SN. However, the contrast between the $\mathrm{SN}$ region and the lensing cluster galaxy is much better at the wavelengths of the [O II] line than in the continuum, and if the $\mathrm{SN}$ were the dominant source of [O II] emission in this $500 \mathrm{pc}$ region, we should detect the variability. We studied the [O II] line at the three different epochs and found no significant change in the [O II] luminosity or peak position at the individual positions, see Table 2 . The lack of variability means either no variation in the $\mathrm{SN}$ [O II] luminosity over this period, or a relative contribution of the $\mathrm{SN}$ to the $[\mathrm{O}$ II] line that is too small to be distinguished with our spectrophotometric precision. The small variation is not surprising, because only $\mathrm{SN}$ with ejecta interacting with circumstellar matter are observed to have clear [O II] emission in their spectra (e.g. Filippenko 1997; Smith et al. 2009b; Pastorello et al. 2015).

\section{Discussion}

Most other galaxies where $\mathrm{Mg}$ II emission is found in absence of absorption are also lensed by foreground clusters and galaxies (e.g. Pettini et al. 2010; Rigby et al. 2014). Similar to these, we find that only some regions of Sp1149 show Mg II emission, while other regions show clear absorption. This means that if the complete galaxy is observed with a single slit, the absorption line would dominate the spectrum. This highlights the importance of spatially resolving galaxies, which is currently only possible at $z>1$ for gravitationally lensed and magnified sources.

The Mg II emission accompanied by a weak Fe II $\lambda 2383 \AA$ absorption line is predicted by the models of Prochaska et al. (2011) and observed by Erb et al. (2012). While most Fe absorption lines are coupled to fine-structure transitions, Fe II $\lambda 2383 \AA$ is only able to fall back to the ground state. This leads to a higher degree of emission line filling, similar to Mg II. However, this also suggests that the other Fe transitions should be clearly visible in the spectra, and it remains unclear why recombination in the $\mathrm{H}$ II regions would affect the resonant Fe II lines differently than the resonant $\mathrm{Mg}$ II lines.

The absence of the Fe II lines at all positions of SN Refsdal indicates that the medium is optically thin, at least for these transitions, and that this is not caused by the exploding SN. The low opacity at the Fe lines agrees with the occurrence of Mg II emission from HII regions, as that would also require an optically thin gas in front of the emitting regions, possibly leaking continuum-ionizing-photons. Assuming $\tau_{\mathrm{Mg} \text { II }}<1$ and $Z=0.2 Z_{\odot}$ limits the column density to $N_{\mathrm{H}}<3 \times 10^{16} \mathrm{~cm}^{-3}$. This provides another indication that the feedback of hot young stars, possibly in combination with previous $\mathrm{SNe}$, has removed most of the cold gas from the environment before SN Refsdal exploded, without forming the shell required for resonant scattering. The early removal of cold gas is in line with predictions of recent feedback models, where young and hot stars dilute the ISM before SN explosions, and therefore provides a first step towards the confirmation of early-stellar feedback.

\section{Summary and conclusions}

We used VLT/MUSE to study the properties of the ISM around the multiply imaged SN Refsdal. We found clear Mg II and weak Fe II* emission, in combination with almost absent Fe II absorption. We determined that the Mg II emission is constrained to the clump surrounding the SN and also found it at the modelpredicted positions in the other two images. This shows that these conditions are not caused by the SN, but are rather the consequence of the young stellar population as a whole. By using the ratio of the [O II] and $\mathrm{Mg}$ II flux, we showed that the ionization parameter is higher than normally seen in H II regions. This can have important consequences for feedback models because our results suggest that stellar feedback needs to be included as well.

We used the [O II] lines to detect possible variation in the luminosity of the SN and thereby constrain the light curve. However, we did not find a significant variability, suggesting that the $\mathrm{SN}$ is either not variable on these timescales or that it is not 
a major contributor to the integrated [O II] flux at the scale resolved by our observations.

Acknowledgements. Based on observations made with the European Southern Observatory Very Large Telescope (ESO/VLT) at Cerro Paranal, under program ID 294.A-5032. The authors thank Patrick Kelly and Pece Podigachoski for useful comments and discussions. This research made use of APLpy, an open-source plotting package for Python hosted at http://aplpy.github.com

\section{References}

Adamo, A., Östlin, G., Bastian, N., et al. 2013, ApJ, 766, 105 Artale, M. C., Tissera, P. B., \& Pellizza, L. J. 2015, MNRAS, 448, 3071 Bacon, R., Accardo, M., Adjali, L., et al. 2012, The Messenger, 147, 4

Ben Zhu, G., Comparat, J., Kneib, J.-P., et al. 2015, ApJ, submitted [arXiv: 1507.07979]

Diego, J. M., Broadhurst, T., Chen, C., et al. 2015, ArXiv e-prints [arXiv: 1504.05953]

Erb, D. K., Quider, A. M., Henry, A. L., \& Martin, C. L. 2012, ApJ, 759, 26 Filippenko, A. V. 1997, ARA\&A, 35, 309

Guseva, N. G., Izotov, Y. I., Fricke, K. J., \& Henkel, C. 2013, A\&A, 555, A90

Hopkins, P. F., Kereš, D., Oñorbe, J., et al. 2014, MNRAS, 445, 581

Karman, W., Caputi, K. I., Grillo, C., et al. 2015, A\&A, 574, A11

Kelly, P. L., Rodney, S. A., Treu, T., et al. 2015, Science, 347, 1123

Kim, C.-G., \& Ostriker, E. C. 2015, ApJ, 802, 99
Kinney, A. L., Bohlin, R. C., Calzetti, D., Panagia, N., \& Wyse, R. F. G. 1993, ApJS, 86, 5

Kornei, K. A., Shapley, A. E., Martin, C. L., et al. 2013, ApJ, 774, 50

Kurk, J., Cimatti, A., Daddi, E., et al. 2013, A\&A, 549, A63

Livermore, R. C., Jones, T., Richard, J., et al. 2012, MNRAS, 427, 688

Livermore, R. C., Jones, T. A., Richard, J., et al. 2015, MNRAS, 450, 1812

Martin, C. L., Shapley, A. E., Coil, A. L., et al. 2012, ApJ, 760, 127

Martizzi, D., Faucher-Giguère, C.-A., \& Quataert, E. 2015, MNRAS, 450, 504

Oguri, M. 2015, MNRAS, 449, L86

Pastorello, A., Wang, X.-F., Ciabattari, F., et al. 2015, MNRAS, submitted [arXiv: 1509.09069]

Pettini, M., Christensen, L., D’Odorico, S., et al. 2010, MNRAS, 402, 2335

Prochaska, J. X., Kasen, D., \& Rubin, K. 2011, ApJ, 734, 24

Rigby, J. R., Bayliss, M. B., Gladders, M. D., et al. 2014, ApJ, 790, 44

Rubin, K. H. R., Prochaska, J. X., Ménard, B., et al. 2011, ApJ, 728, 55

Sharon, K., \& Johnson, T. L. 2015, ApJ, 800, L26

Smith, G. P., Ebeling, H., Limousin, M., et al. 2009a, ApJ, 707, L163

Smith, N., Silverman, J. M., Chornock, R., et al. 2009b, ApJ, 695, 1334

Stinson, G. S., Brook, C., Macciò, A. V., et al. 2013, MNRAS, 428, 129

Tang, Y., Giavalisco, M., Guo, Y., \& Kurk, J. 2014, ApJ, 793, 92

Vasiliev, E. O., Nath, B. B., \& Shchekinov, Y. 2015, MNRAS, 446, 1703

Weiner, B. J., Coil, A. L., Prochaska, J. X., et al. 2009, ApJ, 692, 187

Yuan, T.-T., Kewley, L. J., Swinbank, A. M., Richard, J., \& Livermore, R. C. 2011, ApJ, 732, L14

Yuan, T.-T., Kobayashi, C., \& Kewley, L. J. 2015, ApJ, 804, L14

Zitrin, A., \& Broadhurst, T. 2009, ApJ, 703, L132 